\title{
Off-Normal Solar Optical Property Measurements Using an Integrating Sphere
}

\author{
Collins $^{1,2}$, M., Wright ${ }^{1,3}$, J.L., Kotey ${ }^{1,4}$, N. \\ ${ }^{1}$ Dept. of Mechanical and Mechatronics Engineering, University of Waterloo \\ 200 University Ave W., Waterloo, Ontario, Canada \\ ${ }^{2}$ Corresponding Author \\ E: mcollins@uwaterloo.ca \\ T: 519.888.4567 x33655 \\ $\mathrm{F}: 519.888 .6197$ \\ ${ }^{3}$ E: jlwright@uwaterloo.ca \\ ${ }^{4}$ E: nathankotey@gmail.com
}

Please note that this file contains the final draft version of this technical paper. Minor differences will be found between this version and the final version printed by the publisher. The reader should contact the publisher if the final version, as printed, is preferred.

The final publication is available at Elsevier via http://dx.doi.org/10.1016/j.measurement.2011.09.016 (C) 2012. This manuscript version is made available under the CC-BY-NC-ND 4.0 license

http://creativecommons.org/licenses/by-nc-nd/4.0/ 


\title{
Off-Normal Solar Optical Property Measurements Using an Integrating Sphere
}

\author{
Collins $^{1,2}$, M., Wright ${ }^{1,3}$, J.L., Kotey ${ }^{1,4}$, N. \\ ${ }^{1}$ Dept. of Mechanical and Mechatronics Engineering, University of Waterloo \\ 200 University Ave W., Waterloo, Ontario, Canada \\ ${ }^{2}$ Corresponding Author \\ E: mcollins@uwaterloo.ca \\ T: $519.888 .4567 \times 33655$ \\ $\mathrm{F}: 519.888 .6197$ \\ ${ }^{3}$ E: jlwright@uwaterloo.ca \\ ${ }^{4}$ E: nathankotey@gmail.com
}

\begin{abstract}
Shading devices attached to windows can be used to control solar gain and hence reduce building peak load and annual energy consumption. The performance of a shading device in this regard is strongly dependent on its solar optical properties. The current study discusses a unique measurement technique that was used to obtain off-normal solar optical properties of flat shading materials. The off-normal properties were needed in order to develop solar optical property models both for shading materials and shading devices. These models provide useful input to building peak load calculation and annual energy simulation tools. Special sample holders were designed and fabricated to facilitate measurements using a commercial spectrophotometer with integrating sphere attachment. A combination of theory and companion tests were used to confirm the validity of the approach.
\end{abstract}

Keywords: integrating sphere, spectrophotometer, solar optical, shading 


\subsection{Introduction}

Due to the rising cost of conventional energy sources, there is an increased interest in creating buildings that not only conserve energy, but also respond to the environment in ways that control energy flow into and out of the space in a beneficial way. Building energy consumption constitutes more than $40 \%$ of the total energy consumption in the US and Canada. Improving the thermal performance of windows has great potential in this regard. It is understood that about $25 \%$ of a building's energy consumption can be attributed to windows. Solar gain will generally increase peak cooling load and cooling energy consumption, and decrease heating energy consumption. The ability to control solar gain is especially important for the successful operation of well insulated, energy efficient buildings. Fortunately, shading devices offer a cost effective strategy to actively accept or reject solar gain. Solar gain can be accepted when heating is required and rejected otherwise.

The performance of windows (shaded or not) can be estimated by taking advantage of the fact that there is no appreciable overlap between the solar and longwave radiation bands. This leads to a two-step analysis. First, with knowledge of the solar optical properties of the individual glazing/shading layers, solar radiation models determine the fraction of incident solar radiation directly transmitted and the fraction that is absorbed in each layer. Since the solar incidence angle changes by the hour, the off-normal solar optical properties of the individual layers must be available. The absorbed solar radiation in each layer then serves as a source term in the second step - the heat transfer analysis. A typical building energy simulation (e.g., EnergyPlus [1], ESP-r [2]) and peak cooling loads software (e.g., ASHRAE Toolkit [3]) might include this analysis in its hour-by-hour calculation.

Until recently, the aforementioned analysis had only been applied to unshaded windows. In that scenario, all of the layers are flat, homogenous, and specular. Further, the normal and off-normal solar optical properties of each layer are easily determined, either by experiment, or theory in the case of uncoated glass. In contrast, shading material aren't necessarily flat, homogenous or specular, and it is difficult to determine solar optical properties; normal or off-normal.

The issues of flatness and homogeneity can be accommodated by assuming that the shading layer can be represented by an equivalent homogenous layer that is assigned 
spatially-averaged “effective” optical properties [4]. This approach has been successfully applied to shades such as Venetian blinds [5] and pleated drapery [6].

The fact that many shade materials exhibit both specular and diffuse behaviour adds complexity. When solar radiation is incident on, for example, drapery, some portion of the radiation passes undisturbed through openings in the fabric and the remaining portion is intercepted by the structure of the fabric. The portion of the intercepted radiation that is not absorbed will be scattered and will leave the layer as an apparent reflection or transmission. These scattered components are assumed to be uniformly diffuse [6].

If the structure is simple, analytical approaches can be used to estimate the effective solar optical properties of a fabric. For example, ray-tracing has successfully been employed to determine the normal and off-normal effective properties of insect screens [7]. This approach, however, is limited. In the case of insect screens, the structure is uniform, and the screen material has a quantifiable shape and solar optical properties. Fabrics however, consist of strands of yarn that are woven or knitted. The yarn itself is made up of fibres that are twisted and plied. Strands of yarn can be woven loosely, leaving open areas, or woven tightly with little or no open area. Furthermore, strands of yarn that are loosely twisted and plied could have open areas between the fibres. As a result, the task of representing drapery fabric in a mathematical model is impractical. More importantly, the model requires that the properties and shape of the fabric material (yarn, thread, etc) be known. Obtaining that information is likely not possible, and experimental techniques are required.

One such experimental study was performed by Hunn et al. [8]. They designed an apparatus to measure the bidirectional transmittance and reflectance of fabrics. The measurements revealed the effect of textile properties (openness of weave, fibre cross section and fabric structure) on the distribution of sunlight. This information can be incorporated into a high-level computation model used to predict the bidirectional solar optical properties of the window system $[9,10]$. This experimental method and the associated glazing/shading layer analysis, however, are not well suited to building energy simulation. The experimental approach provides far more detail than is required, and consequently, the complexity and cost (both monetary and time) make the approach restrictive. The required CPU time is also impractical. 
In the current study, a new technique was developed and evaluated for measuring the off-normal properties of flat shading materials. Special sample holders were designed and fabricated to facilitate the measurement of solar optical properties of flat shading materials as a function of incidence angle using an integrating sphere installed in a commercially available spectrophotometer. The integrating sphere is particularly useful because it can resolve the undisturbed and scattered components of transmitted or reflected beam radiation. The sample holders were made from polished aluminum tubes with one end truncated at a known angle, $\theta$ (where $\theta=0$ represents direct normal). The interior surface of each tube was painted black in order to absorb radiation scattered in reflection during a transmittance measurement or scattered in transmission during a reflectance measurement. A similar technique was successfully used by Pettit [11] to measure the off-normal transmittance of specular glazings layers.

Spectral measurements of beam-total and beam-diffuse transmittance, and beam-total reflectance were obtained on fabric samples at incidence angles, $\theta$, ranging from 0 to $60^{\circ}$ [12].

\subsection{Measurement Methodology}

\subsection{Spectrophotometer and Integrating Sphere}

The spectrophotometer used in this study is a double beam, direct ratio recording, rapid scanning instrument. It has a resolution of less than $0.05 \mathrm{~nm}$ for the ultraviolet and visible spectra (UV-VIS) and less than $0.2 \mathrm{~nm}$ for the near infrared spectrum (NIR); a repeatability characteristic of less than $0.025 \mathrm{~nm}$ for UV-VIS and less than $0.1 \mathrm{~nm}$ for NIR. In operation, two detectors are used; a Photomultiplier Tube (PMT) and a lead-sulphide (PbS) photoconductive sensor. The PMT is used in the wavelength range of $0.17<\lambda<0.8 \mu \mathrm{m}$ and the $\mathrm{PbS}$ in the range of $0.8<\lambda<3.3 \mu \mathrm{m}$.

For the purpose of the current investigation the spectrophotometer was operated with an integrating sphere attachment. The integrating sphere is a spherical enclosure with a highly reflective and Lambertian coating. By a combination of geometry and the highly reflective surface, light that enters the sphere becomes uniformly distributed over the entire inner surface, eliminating all directionality from that light, and producing a uniform (or integrated) light field within the sphere. The detectors can then be used to measure the intensity of this light field. The detectors are shielded by baffles so that they do not view the light source as it 
enters the sphere, or its first reflection. Depending on how they are configured, integrating spheres can measure and distinguish between, beam and scattered components of transmitted and reflected radiation. Theory and operating principles can be found in many references (e.g., $[13,14])$.

The integrating sphere used in this study is a $110 \mathrm{~mm}$ diameter sphere with a polytetrafluoroethylene (PTFE) coating. The spectral reflectance characteristic of the PTFE decreases at longer wavelengths and so useful measurements are restricted to the $0.25<\lambda<$ $2.5 \mu \mathrm{m}$ where $\rho_{\mathrm{PTFE}}=0.98$. Nonetheless, this wavelength range includes almost $98 \%$ of the solar spectrum.

It is important to note that the integrating sphere takes advantage of the double beam capability of the spectrophotometer. Both a sample and a reference beam exist, each with its own entry port. These beams, however, are not of equal intensity. The optics upstream of the sphere, and the point of first reflection within the sphere itself, are different. To adjust for this, the system is calibrated, at each wavelength, prior to taking measurements. The calibration procedure varies depending on the type of measurement required, and so each will be described in turn. It is further noted that the double beam capability accounts for changes in sphere response. Placing a sample in the integrating sphere will result in a reduction in signal strength from the detectors. Since both the sample and reference beams are affected similarly, this effect can be factored out.

Calibration for transmittance measurements is performed as follows (Fig. 1). First, a $100 \%$ baseline test is performed (Fig. 1a). To do so, readings are taken with no obstruction in the transmission port, and with a PTFE insert at the reflection port that completes the sphere. The ratio of detector signal strength between the sample beam and the reference beam should ideally be unity. The ratio that is measured, $100_{\lambda}$, becomes a scaling factor. Next, a $0 \%$ baseline is performed (Fig. 1b). Here, the transmission port is blocked using an opaque material, while the PTFE insert remains at the reflectance port. The ratio of detector signal strength would ideally be 0 in this case, and the measured ratio, $Z_{\lambda}$, becomes an offset value. 


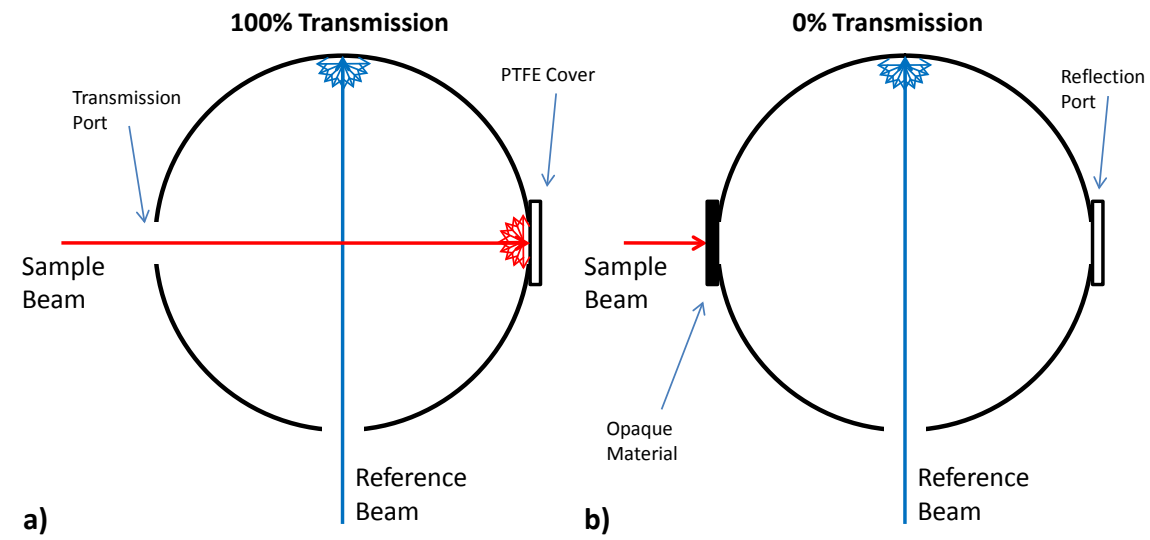

Figure 1: Integrating sphere calibration for transmission tests: a) $100 \%$ transmission, b) $0 \%$ transmission.

To perform a transmittance measurement, $\tau_{\lambda}$, a test sample is mounted over the transmission port (Fig. 2). The ratio of detector signal strength, $S_{\lambda}$, is obtained. Transmittance is then determined using ASTM E903-96 [15]

$$
\tau_{\lambda}=\frac{S_{\lambda}-Z_{\lambda}}{100_{\lambda}-Z_{\lambda}}
$$

This approach can be used to determine both the beam-total transmittance, $\tau_{\lambda, \text { bt }}$, and the beamdiffuse transmittance, $\tau_{\lambda, \text { bd }}$. To obtain beam-total transmittance, $S_{\lambda}$ is measured with the PTFE insert in place at the reflectance port (Fig. 2a). To obtain beam-diffuse transmittance, $\mathrm{S}_{\lambda}$ is measured with the reflectance port open, so that the beam component can pass through the integrating sphere (Fig. 2b). The beam-beam transmittance, $\tau_{\lambda, \text { bb }}$, is the difference between the $\tau_{\lambda, \mathrm{bt}}$ and $\tau_{\lambda, \mathrm{bd}}$ measurements.

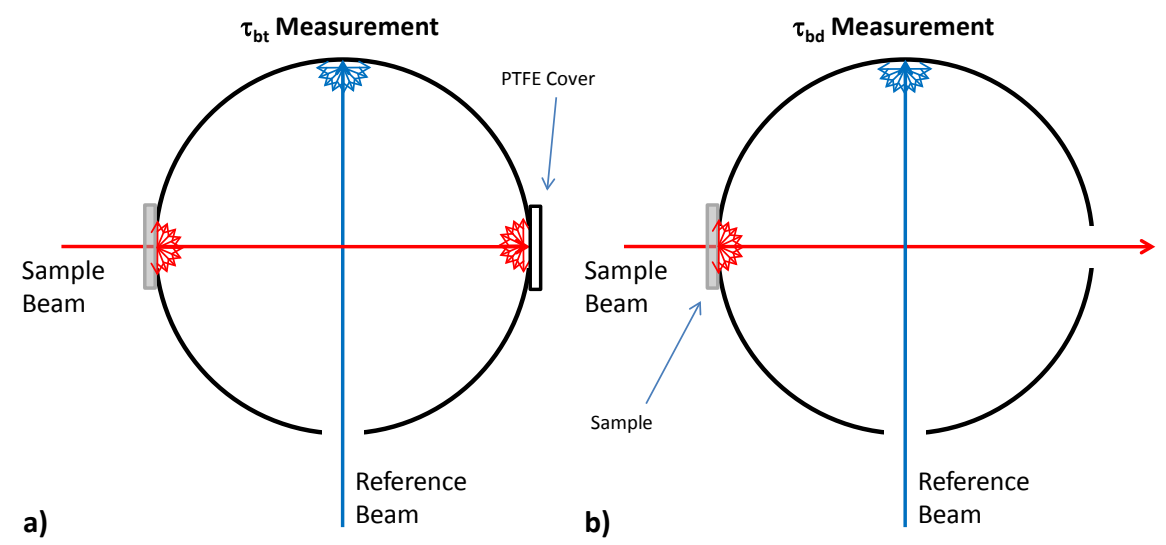

Figure 2: Integrating sphere configuration: a) beam-total transmittance, b) beam-diffuse transmittance. 
Calibration for reflectance measurements differs from that described for transmittance measurements (Fig. 3). Again, a 100\% baseline test is first performed (Fig. 3a). To do so, readings are taken with no obstruction in the transmission port, and with a reflectance standard of known reflectivity, $\rho_{\lambda \text {,ref }}$ covering the reflection port. The ratio of detector signal strength between the sample and reference beams, $100_{\lambda}$, is obtained. The $0 \%$ baseline is also performed (Fig. 3b) with both the transmission and reflectance ports open, allowing the sample beam to pass through the integrating sphere. This process allows for scatter in the sample beam to be calibrated out of the measurement. The ratio of detector signal strength, $\mathrm{Z}_{\lambda}$, is determined.

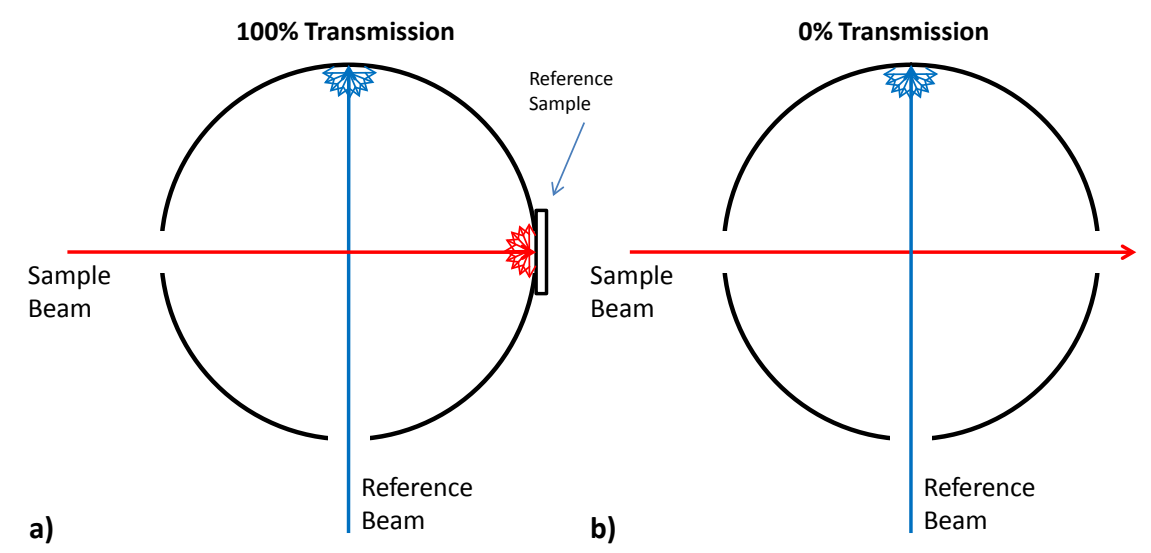

Figure 3: Integrating sphere calibration for reflection tests: a) $100 \%$ transmission, b) $0 \%$ transmission.

To measure reflectance, $\rho_{\lambda}$, tests samples are placed at the reflection port (Fig. 4). The ratio of detector signal strength, $\mathrm{S}_{\lambda}$, is then obtained. The three measurements are used to determine reflectance using Equation (2) [15].

$$
\rho_{\lambda}=\left(\frac{S_{\lambda}-Z_{\lambda}}{100_{\lambda}-Z_{\lambda}}\right) \rho_{\lambda, \text { ref }}
$$

The reflectance measurement can either be beam-total reflectance, $\rho_{\lambda, \mathrm{bt}}$, or beam-diffuse reflectance, $\rho_{\lambda, \text { bd. }}$ This is achieved through the use of a sample positioning cap (that contains the reflectance port). To measure beam-total reflectance, the cap is positioned such that the sample is at a slight angle ( $\theta \approx 3^{\circ}$ in this case) with respect to the sample beam. As a result, any specular reflection, $\rho_{\lambda, \mathrm{bb}}$ is directed to the side of the integrating sphere, and is included in the measurement. To measure beam-diffuse reflectance, the cap is positioned so that incident 
beam is normal to the sample. Any specular reflection will exit the sphere through the transmission port, and will not be included in the measurement. Again, $\rho_{\lambda, \mathrm{bb}}$ is simply the difference between the $\rho_{\lambda, \text { bt }}$ and $\rho_{\lambda, \text { bd }}$ readings.

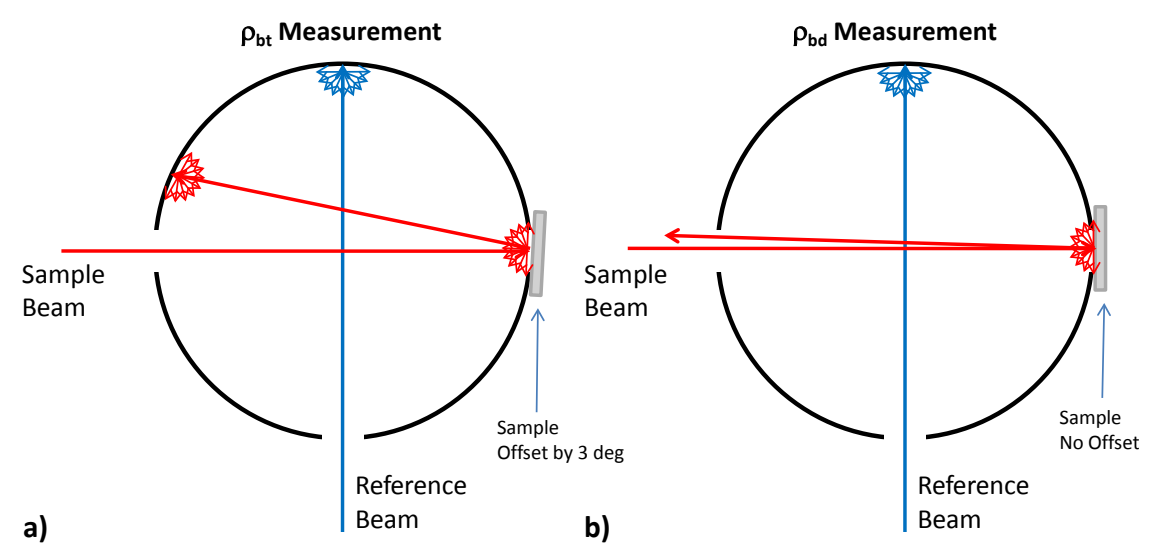

Figure 4: Integrating sphere configuration: a) beam-total reflectance, b) beam-diffuse reflectance.

It is worth noting that, by not factoring in the diffuse or specular nature of the sample during calibration, a measurement error is introduced. Transmission calibration is carried out on what is effectively a purely specular transmitter (i.e., air). The first reflection from the sphere wall is not within the detectors view, and therefore the sample beam must reflect at least twice before it is sensed. When a measurement is performed on a diffusing sample, the detectors sense radiation after only one reflection. The result is that the transmission tends to be over-predicted by a factor of $1 / \rho_{\text {PTFE }}$ (i.e., a $2 \%$ over-prediction of transmittance for diffusing samples). Conversely, reflectance calibration is performed using a diffusing calibration standard. Light striking the calibration standard is sensed by the detector after two reflections. If a specularly reflecting sample is then measured, neither the first reflection (from the sample) nor the second reflection (from the sphere wall) will be sensed by the detectors. The signal is not sensed until after the third reflection. The result is that measurements of specular samples tend to be under-predicted by a factor of $\rho_{\text {PTFE }}$ (i.e., a $2 \%$ under-prediction of reflectivity for specular samples). (See Appendix A for more detail). This error tends to be ignored. Nonetheless, these effects are germane to the test procedure introduced in this work, and are further discussed in a later section. 


\subsection{Adaptation of Integrating Sphere for Off-Normal Measurements}

Sample holders were designed and fabricated to adapt the integrating sphere for measurement of transmittance and reflectance at off-normal incidence (Fig. 5). The sample holders were made from aluminium tubes with one end machined at an angle to permit incidence angles ranging from zero to $60^{\circ}$ in $15^{\circ}$ steps. Adapters were also built to mount sample holders at either the reflection or transmission port. Each sample holder is $40 \mathrm{~mm}$ long with internal and external diameters of 13.75 and $15.75 \mathrm{~mm}$. At the transmission port, the incident beam is rectangular in cross-section with dimensions of $13.44 \mathrm{~mm} \times 11.04 \mathrm{~mm}$. Thus, the diagonal of the beam cross-section is $17.39 \mathrm{~mm}$ which is greater than the internal diameter of the holder. Preliminary investigation showed that measurements were in error by about $2 \%$ because the fixed sample holder tubes were intercepting a small portion of the incident beam. To ensure that the incident beam would pass through the holder without interference a beam reducer was glued to the outer face of the transmission port adapter. The beam reducer is simply a thin plate with a $12.80 \mathrm{~mm}$ diameter hole. Reduction of the incident beam eliminated the aforementioned error.

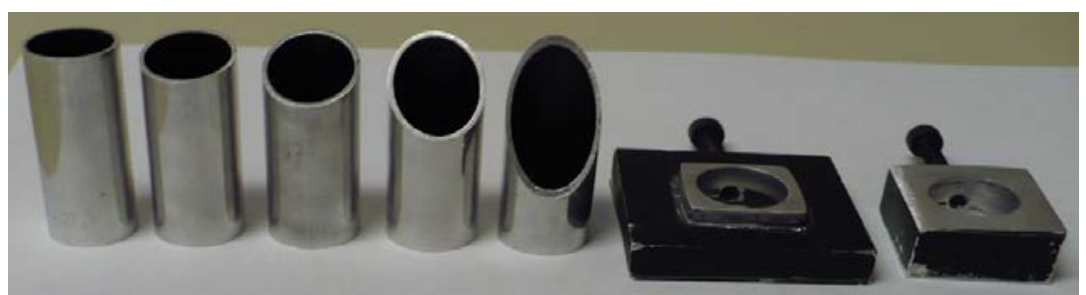

Figure 5: A set of fixed sample holders, transmission and reflection port adapters

Typical configurations for transmittance and reflectance measurements using a fixed sample holder are shown in Figures 6 and 7. In each case the sample holder projected into the sphere a distance of $30 \mathrm{~mm}$. For the integrating sphere used in this study, this projection distance was not enough to place the sample holder within direct view of the detectors. Ideally, the exterior surface of the sample holder should be coated with a highly reflective coating such as PTFE or barium sulphate. Such a surface would have a very low absorptance thereby ensuring that the detector signal strength would not be reduced appreciably. However, it was too expensive to use a PTFE coating, and barium sulphate coating did not adhere well to the sample holder. A more pragmatic way of achieving a highly reflective sample holder was to polish the exterior surface of the sample holder. However, it was not clear what impact the highly specular polished sample holder might have on the measurements. 


\subsection{Off-Normal Transmittance Measurement}

To maintain integrating sphere response characteristics, both the calibration and measurement were performed with fixed sample holders of identical geometry. With the reflection port covered, a sample holder without a sample attached, was mounted at the transmission port as shown in Figure 6. The 100\% baseline readings were taken. The transmission port was blocked and the $0 \%$ baseline readings were taken. A sample was attached to the angled end of an identical sample holder and mounted at the transmission port. With the reflection port closed, spectral readings were taken to obtain $\tau_{\lambda, \text { bt }}(\theta)$ (Fig. 6a). The reflection port was opened and $\tau_{\lambda, \text { bd }}(\theta)$ readings were taken (Fig. 6b).

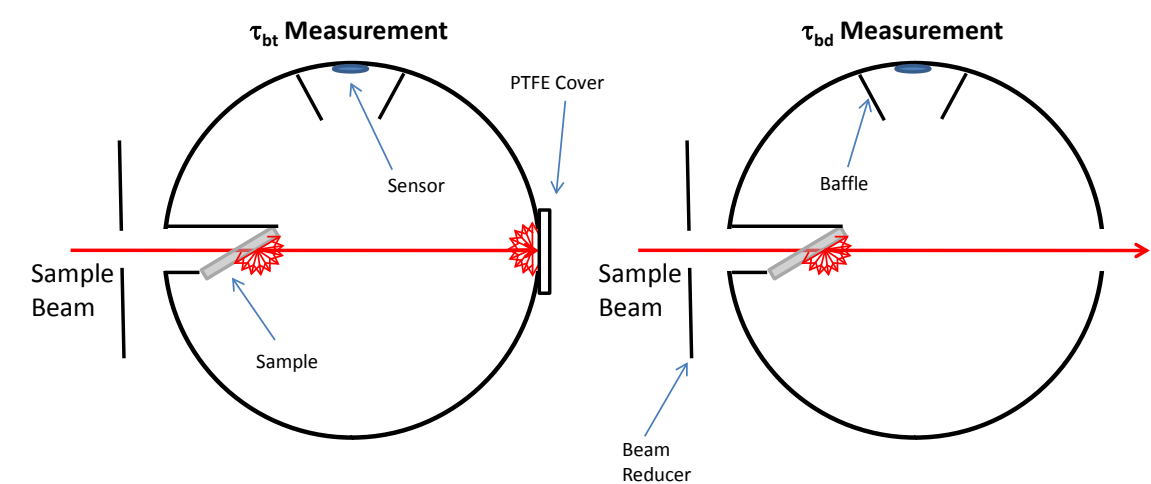

a)

b)

Figure 6: Integrating sphere configuration with fixed sample holder: a) beam-total transmittance, b) beam-diffuse transmittance.

\subsection{Off-Normal Reflectance Measurements}

As with transmittance measurements, to maintain integrating sphere response characteristics, both the calibration and measurement were performed with fixed sample holders of identical geometry. In this case, however, a set of reflectance references had to be fabricated by filling the angled end of sample holders with barium sulphate paste. The paste was pressed against a smooth surface and left to dry. The solar reflectance of barium sulphate $\left(\rho_{\mathrm{BaSu}}=0.96\right)$ was measured to obtain $\rho_{\lambda \text {,ref }}$ (for use in Eqn. (2)). A reflectance reference with an end angle $\theta$ was installed at the reflection port. The transmission adapter was installed at the transmission port to reduce the size of the incident beam. The $100 \%$ baseline was recorded. The reflectance reference was replaced with an open sample holder (same $\theta)$ and the 
$0 \%$ baseline was recorded. Finally, a sample was attached to the angled end of a sample holder (Fig. 7). Beam-total reflectance, $\rho_{\lambda, \text { bt }}$, readings were taken.

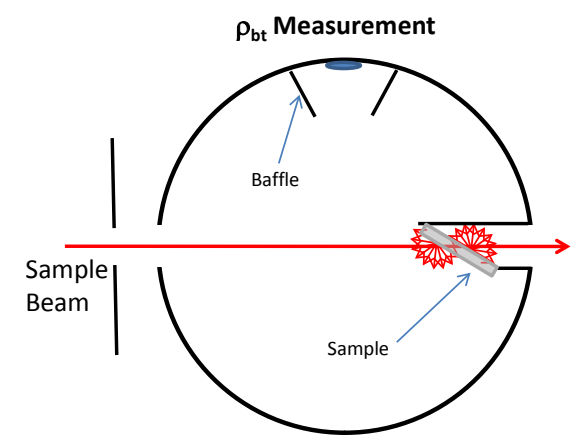

Figure 7: Integrating sphere configuration with fixed sample holder: beam-total reflectance.

\subsection{Calculation of Solar Properties}

Since the aim of the current study was to generate solar (spectrally averaged) optical properties for building energy simulation, no spectral data are presented. The solar optical properties were calculated using the 50-point selected ordinate method described in ASTM E903-96 [15] and using the air-mass 1.5 solar spectrum outlined in ASTM E891-87 [16]. The $\lambda$ subscript is dropped when considering spectrally averaged properties.

\subsection{Additional Considerations}

Consider beam radiation incident on a flat shading material. A portion of the incident beam radiation will pass undisturbed through the openings of the shading material (i.e., fabric, roller blind or insect screen). This constitutes the beam-beam transmittance, $\tau_{\mathrm{bb}}$. The remaining portion is intercepted and undergoes multiple reflections within the structure (e.g., yarn, wires, roller blind material) as well as possible transmission through the structure. The portion of the intercepted radiation that is not absorbed by the material emerges in the forward or backward direction. The portion that emerges in the forward direction constitutes beamdiffuse transmittance, $\tau_{\mathrm{bd}}$, while the portion that emerges in the backward direction is the beam-diffuse reflectance, $\rho_{\mathrm{bd}}$. The word diffuse indicates that these scattered components are assumed to be uniformly distributed when subsequently used in simulation models (e.g. [4]). It was assumed that the shading layers considered do not exhibit specular reflection, $\rho_{\mathrm{bb}} \approx 0$. 
This assumption was later confirmed by experiment. The beam-total reflectance, $\rho_{\mathrm{bt}}$, is therefore equal to the beam-diffuse reflectance, $\rho_{b t}=\rho_{b d}$. The beam-total transmittance, $\tau_{b t}$, is the sum of $\tau_{\mathrm{bb}}$ and $\tau_{\mathrm{bd}}$.

Openness factor is defined as the ratio of the open area to the total area of the sample, viewed normal to the sample. At normal incidence the beam-beam transmittance is a good measure of the openness factor, $A_{o}[12,17]$, i.e., $A_{o}=\tau_{b b}(\theta=0)$.

\subsection{Measurements}

Fabric samples were obtained with the primary aim of locating samples that fit into each of the nine designations outlined by Keyes [17]. He characterised fabrics by yarn colour (yarn reflectance) as dark (D), medium (M), and light (L), and by weave as open (I), semiopen (II), and closed (III). With the exception of designation IIID, samples were obtained for each designation. The thicknesses of the fabric samples ranged from $0.1 \mathrm{~mm}$ to $1.0 \mathrm{~mm}$ and $\mathrm{A}_{\mathrm{o}}$ ranged from 0.01 to 0.34 . The type, colour and openness of each fabric sample are summarized in Table 1.

Table 1: Description of fabric samples

\begin{tabular}{lcc}
\multicolumn{1}{c}{ Type } & Colour & Openness \\
\hline Open Weave, Light Coloured (IL) & White & 0.24 \\
Semi-Open Weave, Light Coloured (IIL) & White & 0.01 \\
Closed Weave, Light Coloured (IIIL) & White & 0.01 \\
Open Weave, Medium Coloured (IM) & Brown & 0.34 \\
Semi-Open Weave, Medium Coloured (IIM) & Green & 0.02 \\
Closed Weave, Medium Coloured (IIIM) & Blue & 0.01 \\
Open Weave, Dark Coloured (ID) & Black & 0.20 \\
Semi-Open Weave, Dark Coloured (IID) & Black & 0.05 \\
\hline
\end{tabular}

The results of measurements taken using the described technique are presented in Table 2. The material characteristics themselves are not germane to the current discussion. The reader is referred to Kotey et al. [12] for a complete discussion of the off-normal properties of drapery fabric. It is noted that the methodology was also applied to roller shades [18] and insect screens [19]. 
Table 2: Summary of measured solar optical properties of fabric samples

\begin{tabular}{ccccccccc}
$\begin{array}{c}\text { Incidence } \\
\begin{array}{c}\text { Angle, } \boldsymbol{\theta} \\
\text { (deg) }\end{array}\end{array}$ & $\begin{array}{c}\text { Open } \\
\text { weave, } \\
\text { light } \\
\text { colored } \\
\text { (IL) }\end{array}$ & $\begin{array}{c}\text { Semi- } \\
\text { open } \\
\text { weave, } \\
\text { light } \\
\text { colored } \\
\text { (IIL) }\end{array}$ & $\begin{array}{c}\text { Closed } \\
\text { weave, } \\
\text { light } \\
\text { colored } \\
\text { (IIIL) }\end{array}$ & $\begin{array}{c}\text { Open } \\
\text { weave, } \\
\text { medium } \\
\text { colored } \\
\text { (IM) }\end{array}$ & $\begin{array}{c}\text { Semi- } \\
\text { open } \\
\text { weave, } \\
\text { medium } \\
\text { colored } \\
\text { (IIM) }\end{array}$ & $\begin{array}{c}\text { Closed } \\
\text { weave, } \\
\text { medium } \\
\text { colored } \\
\text { (IIIM) }\end{array}$ & $\begin{array}{c}\text { Open } \\
\text { weave, } \\
\text { dark } \\
\text { colored } \\
\text { (ID) }\end{array}$ & $\begin{array}{c}\text { Semi- } \\
\text { open } \\
\text { weave, } \\
\text { dark } \\
\text { colored } \\
\text { (IID) }\end{array}$ \\
\hline 0 & 0.24 & 0.02 & 0.01 & 0.33 & 0.02 & 0.01 & 0.20 & 0.04 \\
15 & 0.24 & 0.02 & 0.01 & 0.33 & 0.02 & 0.01 & 0.19 & 0.04 \\
30 & 0.23 & 0.02 & 0.01 & 0.32 & 0.03 & 0.01 & 0.18 & 0.04 \\
60 & 0.18 & 0.01 & 0.00 & 0.27 & 0.01 & 0.00 & 0.11 & 0.03 \\
\hline
\end{tabular}

\begin{tabular}{|c|c|c|c|c|c|c|c|c|}
\hline \multicolumn{9}{|c|}{ Beam-Total Transmittance Measurements } \\
\hline 0 & 0.56 & 0.44 & 0.30 & 0.64 & 0.28 & 0.20 & 0.32 & 0.23 \\
\hline 15 & 0.56 & 0.44 & 0.30 & 0.65 & 0.27 & 0.21 & 0.32 & 0.23 \\
\hline 30 & 0.56 & 0.43 & 0.29 & 0.63 & 0.28 & 0.19 & 0.31 & 0.21 \\
\hline 45 & 0.52 & 0.39 & 0.27 & 0.58 & 0.23 & 0.17 & 0.23 & 0.19 \\
\hline 60 & 0.45 & 0.35 & 0.24 & 0.52 & 0.20 & 0.13 & 0.12 & 0.15 \\
\hline \multicolumn{9}{|c|}{ Beam-Diffuse Transmittance Measurements } \\
\hline 0 & 0.32 & 0.42 & 0.29 & 0.30 & 0.26 & 0.20 & 0.12 & 0.18 \\
\hline 15 & 0.32 & 0.42 & 0.30 & 0.31 & 0.25 & 0.20 & 0.13 & 0.19 \\
\hline 30 & 0.33 & 0.41 & 0.28 & 0.31 & 0.25 & 0.18 & 0.13 & 0.17 \\
\hline 45 & 0.33 & 0.38 & 0.27 & 0.31 & 0.22 & 0.16 & 0.12 & 0.16 \\
\hline 60 & 0.34 & 0.35 & 0.24 & 0.33 & 0.20 & 0.13 & 0.11 & 0.14 \\
\hline \multicolumn{9}{|c|}{ Beam-Total Reflectance Measurements } \\
\hline 0 & 0.42 & 0.56 & 0.67 & 0.23 & 0.31 & 0.37 & 0.15 & 0.21 \\
\hline 15 & 0.43 & 0.56 & 0.68 & 0.24 & 0.33 & 0.37 & 0.15 & 0.21 \\
\hline 30 & 0.44 & 0.58 & 0.69 & 0.26 & 0.33 & 0.39 & 0.16 & 0.21 \\
\hline 45 & 0.48 & 0.62 & 0.71 & 0.30 & 0.36 & 0.42 & 0.18 & 0.24 \\
\hline 60 & 0.54 & 0.65 & 0.74 & 0.37 & 0.38 & 0.46 & 0.21 & 0.26 \\
\hline
\end{tabular}




\subsection{Analysis of Methodology}

Since the technique used to measure off-normal solar properties using the integrating sphere is new, particular attention is devoted to the investigation of both random and systematic uncertainties in the measurements. Both theory and experimentation were considered in the quantification of several possible sources of uncertainty.

\subsection{Measurement Uncertainty}

Uncertainty in standard integrating sphere measurements may be attributed to several factors (ASTM E903-96 [15]). The uncertainty associated with the raw measurements, with or without the integrating sphere, is small. The spectrophotometer was configured to take measurements at $2 \mathrm{~nm}$ intervals. At each wavelength, thirty samples were taken so that the random uncertainty attached to spectral readings was well below \pm 0.001 at a $95 \%$ confidence level. Note that the $0 \%$ and $100 \%$ reference for transmittance as well as the $0 \%$ reference for reflectance are exact quantities. More significant systematic uncertainty is associated with the conversion of spectral data to total solar properties. Systematic uncertainty caused by the choice of solar spectrum was ignored. Since measurements were obtained from the ratio of sample and reference detector signals, correlated uncertainties tend to cancel, reducing the systematic uncertainty in transmittance and reflectance [20]. According to ASTM E903-96 [15], the overall uncertainty (mostly due to systematic uncertainty) associated with measurements $\left(\tau_{\mathrm{bt}}, \tau_{\mathrm{bd}}, \rho_{\mathrm{bt}}\right.$, and $\left.\rho_{\mathrm{bd}}\right)$ taken at normal incidence with an integrating sphere is typically \pm 0.03 at a $95 \%$ confidence level.

If obtained by integrating sphere measurements, $\tau_{\mathrm{bb}}$ is the difference between measurements of $\tau_{\mathrm{bt}}$ and $\tau_{\mathrm{bd}}$.

$$
\tau_{\mathrm{bb}}=\tau_{\mathrm{bt}}-\tau_{\mathrm{bd}}
$$

If $\tau_{\mathrm{bb}}$ is determined in this way, the principle of propagation of uncertainties [21] could be applied to obtain the systematic uncertainty, $\mathrm{B}_{\tau b b}$, as:

$$
\mathrm{B}_{\tau_{\mathrm{bb}}}^{2}=\left(\frac{\partial \tau_{\mathrm{bb}}}{\partial \tau_{\mathrm{bt}}} \mathrm{B}_{\tau_{\mathrm{bt}}}\right)^{2}+\left(\frac{\partial \tau_{\mathrm{bb}}}{\partial \tau_{\mathrm{bd}}} \mathrm{B}_{\tau_{\mathrm{bd}}}\right)^{2}
$$

where $\mathrm{B}_{\tau b t}$ and $\mathrm{B}_{\tau b d}$ are the systematic uncertainties in $\tau_{b t}$ and $\tau_{b d}$, respectively. Equation (4) simplifies to: 


$$
\mathrm{B}_{\tau_{\mathrm{bb}}}^{2}=\mathrm{B}_{\tau_{\mathrm{bt}}}^{2}+\mathrm{B}_{\tau_{\mathrm{bd}}}^{2}
$$

Substituting $\mathrm{B}_{\tau b \mathrm{t}}=\mathrm{B}_{\tau b \mathrm{~d}}=0.03$ into Equation (5) gives $\mathrm{B}_{\tau b \mathrm{~b}}=0.04$. The random uncertainty in $\tau_{\mathrm{bb}}$ is negligibly small and therefore, the overall uncertainty in $\tau_{\mathrm{bb}}$ is equal to the systematic uncertainty at \pm 0.04 .

If relative uncertainties are considered, however, an intriguing result is obtained. The expression for the relative systematic uncertainty, $\mathrm{B}_{\tau b \mathrm{~b}} / \tau_{\mathrm{bb}}$, is given by

$$
\left(\frac{\mathrm{B}_{\tau_{\mathrm{bb}}}}{\tau_{\mathrm{bb}}}\right)^{2}=\left(\frac{\tau_{\mathrm{bt}}}{\tau_{\mathrm{bb}}} \frac{\mathrm{B}_{\tau_{\mathrm{bt}}}}{\tau_{\mathrm{bt}}}\right)^{2}+\left(\frac{\tau_{\mathrm{bd}}}{\tau_{\mathrm{bb}}} \frac{\mathrm{B}_{\tau_{\mathrm{bd}}}}{\tau_{\mathrm{bd}}}\right)^{2}
$$

which simplifies to

$$
\left(\frac{\mathrm{B}_{\tau_{\mathrm{bb}}}}{\tau_{\mathrm{bb}}}\right)^{2}=\left(\frac{\mathrm{B}_{\tau_{\mathrm{bt}}}}{\tau_{\mathrm{bb}}}\right)^{2}+\left(\frac{\mathrm{B}_{\tau_{\mathrm{bd}}}}{\tau_{\mathrm{bb}}}\right)^{2}
$$

Equation (7) reveals that as $\tau_{b b} \rightarrow 0, B_{\tau b b} / \tau_{b b} \rightarrow \infty$. This result raises some concern regarding the integrating sphere method of obtaining reliable values of $\tau_{\mathrm{bb}}$ from measurements of $\tau_{\mathrm{bt}}$ and $\tau_{\mathrm{bd}}$ especially for cases where $\tau_{\mathrm{bb}}$ is small.

Even though the problem of reliable $\tau_{\mathrm{bb}}$ measurements did not present itself, it was decided to investigate the reliability of $\tau_{\mathrm{bb}}$ measurements using an alternate methodology that did not involve the integrating sphere. A rotatable sample holder was made by modifying a Brewster Angle (BA) holder (Fig. 8). An aluminium plate with an aperture where a sample could be mounted was attached to the graduated dial of the BA holder. It enabled $\tau_{\mathrm{bb}}$ measurements over a wide range of incidence angles. The incident beam was simply aligned with the detector such that scattered radiation was excluded. The overall uncertainty associated with this measurement is estimated to be \pm 0.02 at $95 \%$ confidence level. This estimate is considered to be conservative given the fact that majority of the systematic uncertainties in integrating sphere measurements may be attributed to the sphere design. 


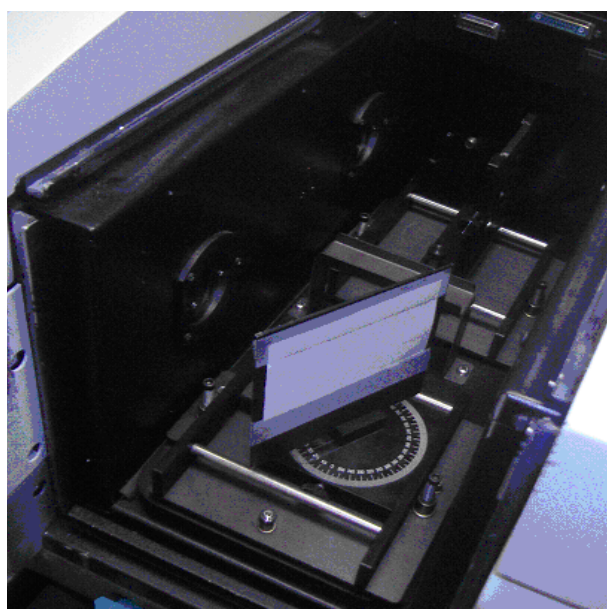

Figure 8: Picture of fabric sample attached to the rotatable sample holder.

A comparison between $\tau_{\mathrm{bb}}$ measurements made with and without the integrating sphere was performed (Table 3). Generally, discrepancies between the two sets of measurements did not exceed \pm 0.04 ; the stated uncertainty associated with integrating sphere measurements. The observed agreement further strengthens the credibility of the measurements procedure. Even though large values of relative error were expected when $\tau_{\mathrm{bb}}$ is small, the results demonstrate that there was no appreciable difference in the measurements.

Table 3: Comparison between beam-beam transmittance measurements of fabric samples with and without the integrating sphere

ID

\begin{tabular}{|c|c|c|c|c|c|c|c|c|c|}
\hline $\begin{array}{l}\text { Incidenc } \\
\text { e Angle }\end{array}$ & $\begin{array}{c}\text { Meas. w/ } \\
\text { Integrating } \\
\text { Sphere }\end{array}$ & $\begin{array}{c}\text { Meas. w/o } \\
\text { Integrating } \\
\text { Sphere }\end{array}$ & Diff & $\begin{array}{c}\text { Meas. w/ } \\
\text { Integrating } \\
\text { Sphere }\end{array}$ & $\begin{array}{c}\text { Meas. w/o } \\
\text { Integrating } \\
\text { Sphere }\end{array}$ & Diff & $\begin{array}{c}\text { Meas. w/ } \\
\text { Integrating } \\
\text { Sphere }\end{array}$ & $\begin{array}{c}\text { Meas. w/o } \\
\text { Integrating } \\
\text { Sphere }\end{array}$ & Diff \\
\hline 0 & 0.20 & 0.23 & 0.03 & 0.33 & 0.32 & -0.01 & 0.24 & 0.26 & 0.02 \\
\hline 15 & 0.19 & 0.23 & 0.04 & 0.33 & 0.32 & -0.01 & 0.24 & 0.25 & 0.01 \\
\hline 30 & 0.18 & 0.21 & 0.03 & 0.32 & 0.31 & -0.01 & 0.23 & 0.23 & 0.00 \\
\hline 45 & 0.11 & 0.14 & 0.03 & 0.27 & 0.27 & 0.00 & 0.18 & 0.18 & 0.00 \\
\hline 60 & 0.01 & 0.02 & 0.01 & 0.19 & 0.18 & -0.01 & 0.11 & 0.09 & -0.02 \\
\hline 70 & & 0.00 & & & 0.06 & & & 0.02 & \\
\hline 80 & & 0.00 & & & 0.00 & & & 0.00 & \\
\hline
\end{tabular}




\subsection{Projection of Sample Holder into the Integrating Sphere}

To establish what impact the projecting sample holders would have on detector signal strength, a rudimentary radiosity analysis was performed (Appendix B). That calculation assumed that the sphere, sample holder, and transmission port each constituted a single surface. For a reflective sample holder projecting $30 \mathrm{~mm}$ into the integrating sphere, that analysis predicted a $20 \%$ reduction in detector signal strength when compared to the same calculation performed without the projecting sample holder.

In conjunction with this analysis, a set of transmission measurements were conducted. Sample holders with two surface finishes were considered representing best and worst case scenarios. These were a highly polished surface with $80 \%$ reflectance and a black painted surface with 5\% reflectance. For each surface finish, a set of sample holders with projected lengths ranging from 5 to $30 \mathrm{~mm}$, in $5 \mathrm{~mm}$ steps, were fabricated. Each sample holder had a $0^{\circ}$ end angle (i.e. cut square).

The integrating sphere was first calibrated in transmission mode. The transmittance of the sphere without a sample holder in place was then measured and was found to be $100 \%$ (as expected). Without recalibration, a sample holder was mounted at the transmission port and a $100 \%$ transmittance measurement taken. Any measurements that deviated from 100\% transmittance would be indicative of relative changes in signal strength between the sample and reference beams. This procedure was repeated for each set of sample holders.

The results of this analysis showed that regardless of the surface finish, the calculated transmission decreased as the projection increased (Fig. 9). The polished sample holder degraded the measurement less than the black painted sample holder. At the maximum projection of $30 \mathrm{~mm}$, the reduction in measurement was $2.8 \%$ for the polished sample holder versus $6.1 \%$ for the black painted sample holder.

It should be emphasized that the bias caused by the presence of sample holders is eliminated by calibration. Accordingly, the total direct-normal transmittance of a number of fabric samples were conducted both using standard measurements procedure, and using a $30 \mathrm{~mm}$ sample holder. In the latter case, the calibration was performed with the sample holder in place. The results of this comparison are shown in Table 4. As can be seen, the difference in all cases is within the experimental uncertainty of the measurement procedure. It is also 
noted that while the preceding analysis was based on transmittance measurements, the same analysis can be applied to reflectance measurements.

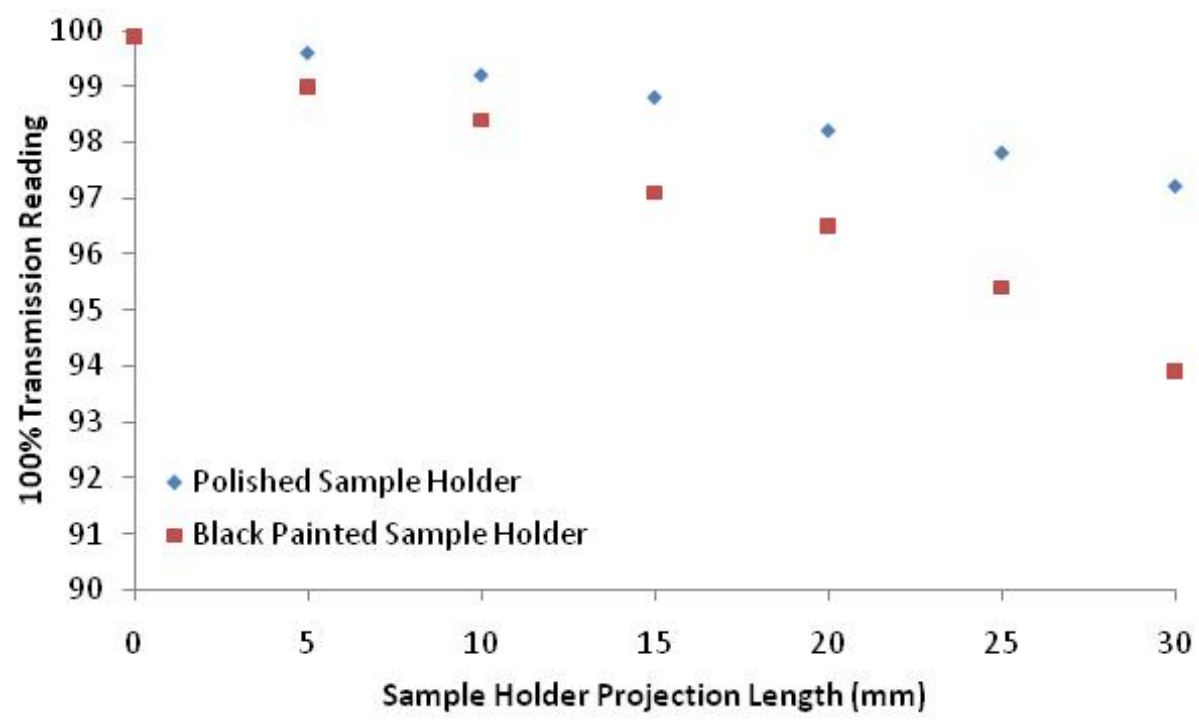

Figure 9: $100 \%$ Transmission readings versus projected length of the sample holder.

Table 4: Comparison of direct-normal solar optical property measurements using standard procedure and sample holders.

\begin{tabular}{|c|c|c|c|c|c|c|c|c|}
\hline leasurement & $\begin{array}{c}\text { Open } \\
\text { weave, } \\
\text { light } \\
\text { colored } \\
\text { (IL) }\end{array}$ & $\begin{array}{l}\text { Semi- } \\
\text { open } \\
\text { weave, } \\
\text { light } \\
\text { colored } \\
\text { (IIL) }\end{array}$ & $\begin{array}{c}\text { Closed } \\
\text { weave, } \\
\text { light } \\
\text { colored } \\
\text { (IIIL) }\end{array}$ & $\begin{array}{l}\text { Open } \\
\text { weave, } \\
\text { medium } \\
\text { colored } \\
\text { (IM) }\end{array}$ & $\begin{array}{c}\text { Semi- } \\
\text { open } \\
\text { weave, } \\
\text { medium } \\
\text { colored } \\
\text { (IIM) }\end{array}$ & $\begin{array}{l}\text { Closed } \\
\text { weave, } \\
\text { medium } \\
\text { colored } \\
\text { (IIIM) }\end{array}$ & $\begin{array}{l}\text { Open } \\
\text { weave, } \\
\text { dark } \\
\text { colored } \\
\text { (ID) }\end{array}$ & $\begin{array}{l}\text { Semi- } \\
\text { open } \\
\text { weave, } \\
\text { dark } \\
\text { colored } \\
\text { (IID) }\end{array}$ \\
\hline
\end{tabular}

\begin{tabular}{ccccccccc}
\hline \multicolumn{7}{c}{ Beam-Total Transmittance Measurements } \\
\hline $\begin{array}{c}\text { With Sample } \\
\text { Holder }\end{array}$ & 0.56 & 0.44 & 0.30 & 0.64 & 0.28 & 0.20 & 0.32 & 0.23 \\
$\begin{array}{c}\text { Standard } \\
\text { Procedure }\end{array}$ & 0.54 & 0.43 & 0.29 & 0.63 & 0.26 & 0.20 & 0.31 & 0.24 \\
& & & & & & & & \\
Difference & -0.02 & -0.01 & -0.01 & -0.01 & -0.02 & 0.00 & -0.01 & 0.01 \\
\hline
\end{tabular}




\subsection{Orientation of the Sample Holder}

It was previously noted that differences between the specular and diffusing nature of samples, when compared to the specular and diffusing nature of the calibration samples, could lead to systematic errors in measured properties (Appendix A). The error here lies with the number of reflections that occur within the sphere before the light is seen by the detector. When compared to a diffusing sample, radiation transmitted or reflected by a specular sample will have one additional reflection from the integrating sphere surface before it can be seen by the detectors. The resulting error is on the order of $\pm 2 \%$. By extension, in any integrating sphere measurement, a systematic uncertainty could arise due to the fact that scattered radiation originating at the sample is not uniformly distributed over the surface of the integrating sphere, and that this non-uniform distribution may be seen by the detectors (i.e. the first reflection seen by the detector has not yet been 'integrated'). Given the typical directional distribution of transmitted or reflected radiation from real materials, the resulting error will be less than the $\pm 2 \%$ quoted above.

When performing measurements with angled and projecting sample holders, the aforementioned problem can become a serious issue. Radiation that originates from a sample will not fully illuminate the surface of the integrating sphere until it has undergone one subsequent reflection from the sphere surface. Should this partially illuminated portion of the sphere include the region viewed by the detectors (called the virtual detector), then the detectors will sense a 'concentrated' first reflection. In its extreme, one could potentially direct a specular reflection directly onto the virtual detector, creating a hot spot. The ratio of sphere surface, $A_{S}$, to illuminated area, $A_{C}$, for each holder is shown in Table 5.

Table 5: $A_{S} / A_{C}$ ratios for each of the angled sample holders.

\begin{tabular}{cccccc} 
& 0 deg & 15 deg & 30 deg & 45 deg & 60 deg \\
\hline $\mathrm{A}_{\mathrm{S}} / \mathrm{A}_{\mathrm{C}}$ & 1.375 & 1.355 & 1.365 & 1.406 & 1.480 \\
\hline
\end{tabular}

In the following discussion, a number of assumptions have been made. Samples are assumed to either be purely specular or purely and uniformly diffuse as appropriate. It is also assumed that reasonable care has been made so that the detectors cannot view the sample or 
projecting sample holder directly. Likewise, care has also been taken to avoid directing any initial specular reflection onto the virtual sensor.

It is noted that a particular notation has been used for convenience. The superscript 'up' refers to a projecting sample holder that faces the sample away from the virtual detector (but not into the view of the detectors). The superscript 'down' refers to a projecting sample holder that faces the sample towards the virtual detector. The locations of the detectors and virtual detector may vary depending on specific integrating sphere geometry.

\subsubsection{Transmission}

Calibration for transmission measurements is described in the Measurement Methodology section of this paper. As mentioned in that section, the ratio of signal strengths from the sample beam and reference beams make up the quantity 100 . Those signal strengths can be represented by the radiosity of the virtual sensor.

First consider the sample beam. It has a flux, $\mathrm{I}_{\text {src }}$, over area, $\mathrm{A}_{\text {src }}$. The $100 \%$ transmission test allows the beam to enter the integrating sphere where it is reflected diffusely from the PTFE coating ( $\rho=\rho_{\text {PTFE}}$ ). This first reflection causes irradiance on the entire sphere surface, $G$, that is not seen by the detectors.

$$
\mathrm{G}=\mathrm{I}_{\mathrm{src}} \frac{\mathrm{A}_{\mathrm{src}}}{\mathrm{A}_{\mathrm{S}}} \rho
$$

The flux, J, leaving any point on the integrating sphere wall after the second reflection is

$$
J=\left(I_{\text {src }} \frac{A_{\text {src }}}{A_{S}} \rho\right) \cdot \rho
$$

This flux is the first and strongest reflection seen by the detector. It follows that after infinite reflections, the total flux sensed by the detector, $\mathrm{J}_{\mathrm{src}}$, is

$$
\begin{gathered}
J_{\mathrm{src}}=\frac{\mathrm{I}_{\mathrm{src}} \mathrm{A}_{\mathrm{src}}}{\mathrm{A}_{\mathrm{S}}} \rho^{2}+\frac{\mathrm{I}_{\mathrm{src}} \mathrm{A}_{\mathrm{src}}}{\mathrm{A}_{\mathrm{s}}} \rho^{3}+\frac{\mathrm{I}_{\mathrm{src}} \mathrm{A}_{\mathrm{src}}}{\mathrm{A}_{\mathrm{S}}} \rho^{4}+\ldots \ldots . . \\
J_{\mathrm{src}}=\frac{\mathrm{I}_{\mathrm{src}} \mathrm{A}_{\mathrm{src}}}{\mathrm{A}_{\mathrm{S}}} \rho^{2}\left(\frac{1}{1-\rho}\right)
\end{gathered}
$$

Similarly, the flux sensed by the detector from the reference beam, $\mathrm{J}_{\text {ref }}$, is

$$
J_{\text {ref }}=\frac{I_{\text {ref }} A_{\text {ref }}}{A_{S}} \rho^{2}\left(\frac{1}{1-\rho}\right)
$$


where the reference beam has a flux, $\mathrm{I}_{\text {ref, }}$ over area, $\mathrm{A}_{\text {ref. }}$ Finally, the ratio 100 is

$$
100=J_{\text {src }} / J_{\text {ref }}=\frac{I_{\text {src }} A_{\text {src }}}{I_{\text {ref }} A_{\text {ref }}}
$$

If a measurement is performed on a specularly transmitting sample, the sample beam behaves similarly to the $100 \%$ transmission test, except that the first reflection is attenuated by the samples transmittance, $\tau_{\text {smp. }}$. Equations 8 and $10 \mathrm{~b}$ become

$$
\begin{gathered}
G=I_{\text {src }} \frac{A_{\text {src }}}{A_{\mathrm{S}}} \tau_{\text {smp }} \rho \\
J_{\text {src }}^{\mathrm{S}}=\frac{I_{\text {src }} A_{\text {src }}}{A_{\mathrm{S}}} \tau_{\text {smp }} \rho^{2}\left(\frac{1}{1-\rho}\right)
\end{gathered}
$$

where $J_{\text {src }}^{S}$ is the flux sensed by the sensor. The ratio $S$ is

$$
\mathrm{S}=\mathrm{J}_{\mathrm{src}}^{\mathrm{S}} / \mathrm{J}_{\text {ref }}=\frac{\mathrm{I}_{\text {src }} \mathrm{A}_{\text {src }} \tau_{\text {smp }}}{\mathrm{I}_{\text {ref }} \mathrm{A}_{\text {ref }}}
$$

Substituting Equations 12 and 15 into Equation 1, and noting that $\mathrm{Z}=0$ shows that the process indeed results in a measurement of $\tau_{\text {smp. }}$.

These two cases demonstrate that the orientation of the sample holder has no impact on the resulting measurement for specular transmittance measurements.

Now consider a measurement of a diffusing sample. The sample holder is oriented such that the sample beam passes through the sample, and is distributed over a section of the sphere with area, $A_{C}$, that does not include the virtual detector. It follows that after infinite reflections, the flux sensed by the detector, $\mathrm{J}_{\text {src }}^{\text {D,up }}$, is:

$$
\begin{gathered}
J_{\text {src }}^{\text {D,up }}=\frac{I_{\text {src }} A_{\text {src }} \tau_{\text {smp }}}{A_{C}} \rho \frac{A_{C}}{A_{\text {s }}} \rho+\frac{I_{\text {src }} A_{\text {src }} \tau_{\text {smp }}}{A_{C}} \rho \frac{A_{C}}{A_{S}} \rho^{2}+\frac{I_{\text {src }} A_{\text {src }} \tau_{\text {smp }}}{A_{C}} \rho \frac{A_{C}}{A_{s}} \rho^{3}+\ldots \ldots . \\
J_{\text {src }}^{\text {D,up }}=\frac{I_{\text {src }} A_{\text {src }} \tau_{\text {smp }}}{A_{S}} \rho^{2}\left(\frac{1}{1-\rho}\right)
\end{gathered}
$$

and

$$
\mathrm{S}=\mathrm{J}_{\text {src }}^{\mathrm{D} \text {,up }} / \mathrm{J}_{\text {ref }}=\frac{\mathrm{I}_{\text {src }} \mathrm{A}_{\text {src }} \tau_{\text {smp }}}{\mathrm{I}_{\text {ref }} \mathrm{A}_{\text {ref }}}
$$

Substituting Equations 12 and 17 into Equation 1, and with $\mathrm{Z}=0$, will again show that the process indeed results in a measurement of $\tau_{\mathrm{smp}}$. The orientation of the sample holder has 
caused an additional reflection in the sample measurement, thereby resulting in an unbiased measurement.

If the same process, however, was carried out on a diffusing sample that illuminates the virtual detector, a different result is obtained.

$$
\begin{gathered}
J_{\text {src }}^{\text {,down }}=\frac{I_{\text {src }} A_{\text {src }} \tau_{\text {smp }}}{A_{C}} \rho+\frac{I_{\text {src }} A_{\text {src }} \tau_{\text {smp }}}{A_{C}} \rho \frac{A_{C}}{A_{S}} \rho+\frac{I_{\text {src }} A_{\text {src }} \tau_{\text {smp }}}{A_{C}} \rho \frac{A_{C}}{A_{S}} \rho^{2}+\ldots \ldots . . \\
J_{\text {src }}^{\text {D,down }}=\frac{I_{\text {src }} A_{\text {src }} \tau_{\text {smp }}}{A_{S}} \rho\left(\frac{A_{S}}{A_{C}}+\frac{\rho}{1-\rho}\right)
\end{gathered}
$$

and

$$
\mathrm{S}=\mathrm{J}_{\text {src }}^{\mathrm{D} \text {,down }} / \mathrm{J}_{\text {ref }}=\frac{\mathrm{I}_{\mathrm{src}} \mathrm{A}_{\text {src }} \tau_{\text {smp }}}{\mathrm{I}_{\text {ref }} \mathrm{A}_{\text {ref }}}\left[\frac{\mathrm{A}_{\mathrm{S}}}{\mathrm{A}_{\mathrm{C}}} \frac{1-\rho}{\rho}+1\right]
$$

Substituting Equations 12 and 19 into Equation 1, with $\mathrm{Z}=0$, and using representative values of $\rho=0.98$ and $A_{S} / A_{C}$ from Table 5 , shows that the value of $\tau_{\text {smp }}$ will be over-predicted by about 3\% for all of the angles considered.

When measuring diffusing samples, it is obvious that efforts should be made so that the virtual detector is not illuminated by the sample itself. If this can be achieved, the measurement can be obtained without bias. Avoiding illumination of the virtual detector, however, is not always possible. If it cannot be avoided it is recommended that the tube be made as short as possible such that the ratio $A_{S} / A_{C}$ approaches unity. In this way, the error approaches what is normally observed (and ignored) by traditional measurement techniques.

It is noted that when obtaining beam-total measurements for Table 2, neither of these guidelines were followed. For measurements at 45 and 60 deg. The sample holder should have been oriented to avoid illuminating the virtual detector. As such, these results should be on the order of $3 \%$ too large. Consider sample IIL; from Table 2, that sample has a beam-total transmittance of 0.39 for a 45 deg incidence angle. When re-measured with the angled sample holder facing away from the virtual detector, the beam-total transmission was 0.37 . The latter measurement is considered more accurate. For the remaining angles, illuminating the virtual sensor was unavoidable, and so the tubes should have been cut shorter to reduce $A_{S} / A_{C}$. In theory, this could have reduced the measurement error from about 3\% to about $2 \%$ too high. Even so, that the predicted bias in these readings is well within the quoted accuracy of the 
measurements $( \pm 0.03)$, and therefore no effort was made to re-measure any of the values presented in Table 2.

\subsubsection{Reflection}

Calibration for reflectivity measurements is described in the Measurement Methodology section of this paper. As mentioned in that section, the ratio of signal strengths from the sample beam and reference beams make up the quantity 100. Those signal strengths can be represented by the radiosity of the virtual sensor.

First consider the sample beam. It has an intensity, $\mathrm{I}_{\text {src }}$, and area, $\mathrm{A}_{\mathrm{src}}$. The $100 \%$ transmission test allows the beam to enter the integrating sphere where it is reflected diffusely from a calibrated reference sample (with reflectivity $\rho_{\text {ref }}$ ). In this case, the irradiance, $G$, falls on a portion of the sphere, $A_{C}$, that does not include the virtual detector.

$$
\mathrm{G}=\mathrm{I}_{\mathrm{src}} \frac{\mathrm{A}_{\mathrm{src}}}{\mathrm{A}_{\mathrm{C}}} \rho_{\text {ref }}
$$

The flux, J, leaving the illuminated portion of the sphere wall after the second reflection is

$$
J=\left(I_{\text {src }} \frac{A_{\text {src }}}{A_{C}} \rho_{\text {ref }}\right) \cdot \rho
$$

This flux is also not viewed by the detector, but is viewed in subsequent reflections. The total flux sensed by the detector, $\mathrm{J}_{\text {src }}^{\mathrm{up}}$, is

$$
\begin{gathered}
J_{\text {src }}^{\text {up }}=\frac{I_{\text {src }} A_{\text {src }}}{A_{S}} \rho_{\text {ref }} \rho^{2}+\frac{I_{\text {src }} A_{\text {src }}}{A_{S}} \rho_{\text {ref }} \rho^{3}+\frac{I_{\text {src }} A_{\text {src }}}{A_{s}} \rho_{\text {ref }} \rho^{4}+\ldots \ldots . . \\
J_{\text {src }}^{\text {up }}=\frac{I_{\text {src }} A_{\text {src }}}{A_{S}} \rho_{\text {ref }} \rho^{2}\left(\frac{1}{1-\rho}\right)
\end{gathered}
$$

The reference beam, however, does not strike an angled sample holder. After its first reflection, it is distributed evenly over the sphere. Following a similar methodology, the flux sensed by the sensor from the reference beam, $\mathrm{J}_{\text {ref, }}$ is still given by Equation 11 , and the ratio 100 is

$$
100=\frac{J_{\text {src }}^{\text {up }}}{J_{\text {ref }}}=\frac{I_{\text {src }} A_{\text {src }} \rho_{\text {ref }}}{I_{\text {ref }} A_{\text {ref }}}
$$


If a measurement is now performed on a diffusely reflecting sample, the sample beam behaves like the $100 \%$ transmission test, except that the first reflection occurs with the samples reflectivity, $\rho_{\text {smp. }}$. Equations 20 and $22 \mathrm{~b}$ respectively become

$$
\begin{gathered}
G=I_{\text {src }} \frac{A_{\text {src }}}{A_{C}} \rho_{\text {smp }} \\
J_{\text {src }}^{\text {D,up }}=\frac{I_{\text {src }} A_{\text {src }}}{A_{\mathrm{S}}} \rho_{\text {smp }} \rho^{2}\left(\frac{1}{1-\rho}\right)
\end{gathered}
$$

where $J_{\text {src }}^{\text {D,up }}$ is the flux sensed by the detector. The ratio $S$ becomes

$$
\mathrm{S}=\mathrm{J}_{\text {src }}^{\text {D,up }} / \mathrm{J}_{\text {ref }}=\frac{I_{\text {src }} A_{\text {src }} \rho_{\text {smp }}}{I_{\text {ref }} A_{\text {ref }}}
$$

Substituting Equations 23 and 26 into Equation 2, and with $Z=0$, the process indeed results in a measurement of $\rho_{\text {smp. }}$.

Now assume that radiation from the calibration sample did illuminate the virtual detector. In this case, $G$ (Eq. 20), falls on a portion of the sphere, $A_{C}$, that does include the virtual detector. Therefore, the flux, J (Eq. 21), leaving the illuminated portion of the sphere wall after the second reflection becomes the first reflection sensed by the detector. This reflection is spread evenly over the entire sphere, and it follows that after infinite reflections, the flux sensed by the detector, $J_{\text {src }}^{\text {down }}$, is

$$
\begin{gathered}
J_{\text {src }}^{\text {down }}=\frac{I_{\text {src }} A_{\text {src }}}{A_{C}} \rho_{\text {ref }} \rho+\frac{I_{\text {src }} A_{\text {src }}}{A_{S}} \rho_{\text {ref }} \rho^{2}+\frac{I_{\text {src }} A_{\text {src }}}{A_{s}} \rho_{\text {ref }} \rho^{3}+\ldots . . . \\
J_{\text {src }}^{\text {down }}=\frac{I_{\text {src }} A_{\text {src }}}{A_{S}} \rho_{\text {ref }} \rho\left(\frac{A_{s}}{A_{C}}+\frac{\rho}{1-\rho}\right)
\end{gathered}
$$

$\mathrm{J}_{\text {ref }}$ is still given by Equation 11, and the ratio 100 is

$$
100=J_{\text {src }}^{\text {down }} / J_{\text {ref }}=\frac{I_{\text {src }} A_{\text {src }} \rho_{\text {ref }}}{I_{\text {ref }} A_{\text {ref }} \rho} \frac{\left(\frac{A_{s}}{A_{C}}+\frac{\rho}{1-\rho}\right)}{\left(\frac{1}{1-\rho}\right)}
$$

If a measurement is now performed on a diffusely reflecting sample, the sample beam behaves like the $100 \%$ transmission test, except that the first reflection occurs with the samples reflectivity, $\rho_{\text {smp. }}$. Equation $27 \mathrm{~b}$ becomes 


$$
J_{\text {src }}^{\text {D,down }}=\frac{I_{\text {src }} A_{\text {src }}}{A_{S}} \rho_{\text {smp }} \rho\left(\frac{A_{S}}{A_{C}}+\frac{\rho}{1-\rho}\right)
$$

and

$$
\mathrm{S}=\mathrm{J}_{\text {src }}^{\mathrm{D} \text { down }} / \mathrm{J}_{\text {ref }}=\frac{I_{\text {src }} A_{\text {src }} \rho_{\text {smp }}}{I_{\text {ref }} A_{\text {ref }} \rho} \frac{\left(\frac{A_{S}}{A_{C}}+\frac{\rho}{1-\rho}\right)}{\left(\frac{1}{1-\rho}\right)}
$$

Substituting Equations 28 and 30 into Equation 2, and with $\mathrm{Z}=0$, the process indeed results in a measurement of $\rho_{\text {smp. }}$.

The orientation of the sample holder has no impact on the measurement of diffuse reflectance, so long as the calibration and measurement are performed with the same sample holder orientation.

Now consider a specularly reflecting sample in any orientation. When the sample beam reflects from the sample it re-encounters the sphere over a small area that is not located on the virtual detector. This reflection is distributed evenly over the sphere. It follows that after infinite reflections, the flux sensed by the detector, $\mathrm{J}_{\mathrm{src}}^{\mathrm{s}}$, is

$$
\begin{gathered}
J_{\text {src }}^{S}=\frac{I_{\text {src }} A_{\text {src }} \rho_{\text {smp }}}{A_{S}} \rho^{2}+\frac{I_{\text {src }} A_{\text {src }} \rho_{\text {smp }}}{A_{S}} \rho^{3}+\frac{I_{\text {src }} A_{\text {src }} \rho_{\text {smp }}}{A_{S}} \rho^{4}+\ldots \ldots . . \\
J_{\text {src }}^{S}=\frac{I_{\text {src }} A_{\text {src }} \rho_{\text {smp }}}{A_{S}} \rho^{2}\left(\frac{1}{1-\rho}\right)
\end{gathered}
$$

and

$$
\mathrm{S}=\mathrm{J}_{\text {src }}^{\mathrm{S}} / \mathrm{J}_{\text {ref }}=\frac{\mathrm{I}_{\text {src }} \mathrm{A}_{\text {src }} \rho_{\text {smp }}}{\mathrm{I}_{\text {ref }} A_{\text {ref }}}
$$

If the calibration was performed such that the virtual sensor was not illuminated, we would substitute Equations 23 and 32 into Equation 2. With $\mathrm{Z}=0$, the process indeed results in a measurement of $\rho_{\text {smp. }}$.

If the calibration were performed such that the virtual sensor was not illuminated, we would substitute Equations 28 and 32 into Equation 2. Here

$$
\rho_{\mathrm{smp}} \neq \frac{\rho_{\mathrm{smp}}\left(\frac{\rho}{1-\rho}\right)}{\left(\frac{A_{\mathrm{s}}}{A_{\mathrm{C}}}+\frac{\rho}{1-\rho}\right)}
$$


Using Equation (33) with representative values of $\rho=0.98$ and $A_{S} / A_{C}$ from Table 5, shows that the value of $\rho_{\text {smp }}$ will be under-predicted by about $3 \%$ for all of the angles considered.

When measuring specular samples, it is obvious that efforts should be made during calibration so that the virtual detector is not illuminated by the calibration sample itself. If this can be achieved, the measurement can be obtained without bias. Avoiding illumination of the virtual detector, however, is not always possible. If it cannot be avoided it is recommended that the tube be made as short as possible such that the ratio $A_{S} / A_{C}$ approaches unity. In this way, the error approaches what is normally observed (and ignored) by traditional measurement techniques.

These calculations can be tested for diffusing samples. From Table 2, the beam-total reflectance of sample IIL was originally measured to be 0.62 at $45 \mathrm{deg}$. The system was calibrated with the reflectance standard facing upward, and then a sample was measured facing upward and downward. The system was then recalibrated with the reference tile facing downward, and upward and downward measurements performed again. The results of this exercise are shown in Table 6. It is noted that while all of the errors are within the quoted accuracy of the measurements $( \pm 0.03)$. Even so, the results lend some credence to the calculations.

Table 6: Effects of Sample Orientation on Measurements. Sample IIL at 45 deg Incident Angle

\begin{tabular}{ccccc}
\hline Calibration Orientation & upwards & downwards & upwards & downwards \\
Sample Orientation & upwards & downwards & downwards & upwards \\
Measurement & 0.62 & 0.63 & 0.65 & 0.61 \\
Measurement Error & $0.0 \%$ & $1.6 \%$ high & $4.8 \%$ high & $1.6 \%$ low \\
Predicted Error & $0.0 \%$ & $0.0 \%$ & $2.8 \%$ high & $2.8 \%$ low \\
\hline
\end{tabular}

\subsection{Conclusions}

A novel technique has been developed to measure the off-normal solar optical properties of flat shading materials by adapting an integrating sphere originally designed to measure solar optical properties at normal incidence. Measurements were taken at varying incidence angles for a wide variety of flat shading materials (i.e., fabrics, roller blinds and insect screens). The measurements include beam and diffuse components. The solar properties obtained at off-normal incidence can be used to develop models on the basis of similar 
properties measured at normal incidence. These off-normal property models serve as useful input to building peak load calculation and annual energy simulation tools.

The technique involved the design and fabrication of special sample holders that were attached to an integrating sphere that is used in conjunction with a spectrophotometer. Although calibration accounts for the presence of the tube inserts, by making them black on the inside, and reflective on the outside, signal strength is maximized (and potential errors are reduced). For reflectivity measurements, the fabrication of reflectance standards is necessary.

To employ this technique, the operator must understand the geometry of their integrating sphere, including the location of the detectors and the virtual detector. By following a few rules, the errors inherent in the technique can be reduced or eliminated. First, never locate the sample within direct view of the detectors. Second, an attempt should be made to design a particular holder such that the radiation leaving the sample does not irradiate the virtual detector. If this is not possible, the sample should illuminate as much of the sphere as possible. Finally, the operator should, for both the calibration and measurement, be aware of how many reflections occur before the detector senses the radiation, and should try to avoid a mismatch between these numbers. For the most part, this is not a problem when measuring transmittance of a specular sample, or reflectance of a diffuse sample. If performing a transmittance measurements on a diffuse sample, or reflectance measurements of a specular sample, orienting the tube upwards may eliminate this problem. For samples that reflect or transmit both diffusely and specularly, some judgement is required.

\section{Acknowledgments}

Financial support in terms of a Natural Sciences and Engineering Research Council (NSERC) Postdoctoral Fellowship to N.A. Kotey and NSERC Discovery Grant funds is gratefully acknowledged. 


\section{Nomenclature}

\section{Greek Letters}

$\rho$

$\tau$

$\theta$

$\lambda$

\section{Symbols}

$\mathrm{A}_{0}$

A

B

G

I

J

S

Z

100

Subscripts

$\infty$

bb

bd

bt

C

ref

$S$

smp

src

Superscripts

down

up

D

S reflectance

transmittance

incidence angle, truncated angle

wavelength

openness factor

surface area

systematic uncertainty

irradiance

incident radiation

radiosity

unadjusted spectrophotometer reading

$0 \%$ baseline spectrophotometer reading

$100 \%$ baseline spectrophotometer reading

related to infinite sum of series

beam-to-beam solar optical property

beam-to-diffuse solar optical property

beam-to-total solar optical property

portion of integrating sphere

related to reference

integrating sphere

related to sample

related to source

facing toward the virtual detector

facing away from the virtual detector

diffuse

specular 


\section{Appendix A: Integrating Sphere Errors due to Specular-Diffuse Mismatch of Sample and Calibration Materials. ${ }^{1}$}

\section{Transmission:}

Calibration for transmission measurements is described in the Measurement Methodology section of the paper. As mentioned in that section, the ratio of detector signal strengths from the sample beam and reference beams make up the quantity 100. Those signal strengths correspond to the radiosity of the part of sphere surface that is viewed by the detector (i.e., the virtual detector).

First consider the sample beam. It has an intensity, $\mathrm{I}_{\text {src }}$, and area, $\mathrm{A}_{\text {src }}$. The $100 \%$ transmission test allows the beam to enter the integrating sphere where it is reflected diffusely from the PTFE coating $\left(\rho=\rho_{\text {PTFE}}\right)$. The irradiance caused by this first reflection, $G$, is uniformly distributed over the entire sphere area.

$$
\mathrm{G}=\mathrm{I}_{\mathrm{src}} \frac{\mathrm{A}_{\text {src }}}{\mathrm{A}_{\mathrm{S}}} \rho
$$

The flux, J, leaving any point on the integrating sphere wall after the second reflection is

$$
J=G \rho=\left(I_{\text {src }} \frac{A_{\text {src }}}{A_{S}} \rho\right) \cdot \rho
$$

This flux is the first and strongest reflection seen by the detector. It follows that after infinite reflections, the total flux sensed by the detector, $\mathrm{J}_{\mathrm{src}}$, is

$$
\begin{gathered}
J_{\text {src }}=\frac{I_{\text {src }} A_{\text {src }}}{A_{s}} \rho^{2}+\frac{I_{\text {src }} A_{\text {src }}}{A_{s}} \rho^{3}+\frac{I_{\text {src }} A_{\text {src }}}{A_{s}} \rho^{4}+\ldots \ldots . \\
J_{\text {src }}=\frac{I_{\text {src }} A_{\text {src }}}{A_{s}} \rho^{2}\left(\frac{1}{1-\rho}\right)
\end{gathered}
$$

Similarly, the flux sensed by the detector from the reference beam, $\mathrm{J}_{\text {ref }}$, is

$$
J_{\text {ref }}=\frac{I_{\text {ref }} A_{\text {ref }}}{A_{S}} \rho^{2}\left(\frac{1}{1-\rho}\right)
$$

where the reference beam has an intensity, $I_{\text {ref }}$, and area, $A_{\text {ref. }}$ The ratio 100 is therefore given by

$$
100=\mathrm{J}_{\text {src }} / \mathrm{J}_{\text {ref }}=\frac{\mathrm{I}_{\text {src }} \mathrm{A}_{\text {src }}}{\mathrm{I}_{\text {ref }} \mathrm{A}_{\text {ref }}}
$$

If a measurement is performed on a specularly transmitting sample, the sample beam behaves similarly to the $100 \%$ transmission test, except that the first reflection is attenuated by the samples transmissivity, $\tau_{\text {smp. }}$ Equations A1 and A3b respectively become

$$
\begin{gathered}
\mathrm{G}=\mathrm{I}_{\mathrm{src}} \frac{\mathrm{A}_{\mathrm{src}}}{\mathrm{A}_{\mathrm{S}}} \tau_{\mathrm{smp}} \rho \\
\mathrm{J}_{\mathrm{src}}^{\mathrm{S}}=\frac{\mathrm{I}_{\mathrm{src}} \mathrm{A}_{\mathrm{src}}}{\mathrm{A}_{\mathrm{S}}} \tau_{\mathrm{smp}} \rho^{2}\left(\frac{1}{1-\rho}\right)
\end{gathered}
$$

where $\mathrm{J}_{\text {src }}^{\mathrm{s}}$ is the flux sensed by the detector from the sample beam after passing a specularly transmitting material.

The ratio $\mathrm{S}$ becomes

\footnotetext{
${ }^{1}$ For the purposes of this discussion, the $\lambda$ subscript has been dropped.
} 


$$
\mathrm{S}=\mathrm{J}_{\text {src }}^{\mathrm{S}} / \mathrm{J}_{\text {ref }}=\frac{\mathrm{I}_{\text {src }} \mathrm{A}_{\text {src }} \tau_{\text {smp }}}{\mathrm{I}_{\text {ref }} \mathrm{A}_{\text {ref }}}
$$

Substituting Equations A5 and A8 into Equation 1, and with that $\mathrm{Z}=0$, shows that the process indeed results in a measurement of $\tau_{\text {smp. }}$.

If a measurement is performed on a diffusely transmitting sample, however, an interesting result is obtained. As the sample beam passes through the sample, it is distributed over the entire surface of the sphere. It follows that after infinite reflections, the flux sensed by the detector from the sample beam after passing a diffusely transmitting material, $\mathrm{J}_{\mathrm{src}}^{\mathrm{D}}$, is

$$
\begin{aligned}
& \mathrm{J}_{\mathrm{src}}^{\mathrm{D}}=\frac{\mathrm{I}_{\mathrm{src}} \mathrm{A}_{\mathrm{src}} \tau_{\mathrm{smp}}}{\mathrm{A}_{\mathrm{S}}} \rho+\frac{\mathrm{I}_{\mathrm{src}} \mathrm{A}_{\mathrm{src}} \tau_{\mathrm{smp}}}{\mathrm{A}_{\mathrm{S}}} \rho^{2}+\frac{\mathrm{I}_{\mathrm{src}} \mathrm{A}_{\mathrm{src}} \tau_{\mathrm{smp}}}{\mathrm{A}_{\mathrm{S}}} \rho^{3}+\ldots \ldots . \\
& \mathrm{J}_{\mathrm{src}}^{\mathrm{D}}=\frac{\mathrm{I}_{\mathrm{src}} \mathrm{A}_{\mathrm{src}} \tau_{\mathrm{smp}}}{\mathrm{A}_{\mathrm{S}}} \rho\left(\frac{1}{1-\rho}\right)
\end{aligned}
$$

and

$$
\mathrm{S}=\mathrm{J}_{\mathrm{src}}^{\mathrm{D}} / \mathrm{J}_{\text {ref }}=\frac{\mathrm{I}_{\mathrm{src}} \mathrm{A}_{\mathrm{src}} \tau_{\text {smp }}}{\mathrm{I}_{\text {ref }} \mathrm{A}_{\text {ref }} \rho}
$$

Substituting Equations A5 and A10 into Equation 1, and with $\mathrm{Z}=0$, shows that an inequality exists. The estimation of $\tau_{\mathrm{smp}}$ is actually $\tau_{\mathrm{smp}} / \rho$, and the calculation will overpredict by $2 \%$ (of the reading, not of full scale). By extension, measurements on a sample that is partially diffusing will have up to $2 \%$ error; depending on the distribution of the transmitted light.

\section{Reflection:}

Calibration for reflectivity measurements is described in the Measurement Methodology section of the paper. As mentioned in that section, the ratio of detector signal strengths from the sample beam and reference beams make up the quantity 100. Those signal strengths can be represented by the radiosity emitted from the virtual detector.

First consider the sample beam. It has a flux, $I_{\text {src }}$, and area, $A_{\text {src }}$. The $100 \%$ transmission test allows the beam to enter the integrating sphere where it is reflected diffusely from a calibrated reference sample (with reflectivity $\rho_{\text {ref }}$ ). The irradiance caused by this first reflection from the reference sample, $\mathrm{G}$, is uniformly distributed over the entire sphere area.

$$
\mathrm{G}=\mathrm{I}_{\mathrm{src}} \frac{\mathrm{A}_{\text {src }}}{\mathrm{A}_{\mathrm{S}}} \rho_{\text {ref }}
$$

The flux, J, leaving any point on the sphere wall after second reflection is given by

$$
\mathrm{J}=\mathrm{G} \rho=\left(\mathrm{I}_{\mathrm{src}} \frac{\mathrm{A}_{\text {src }}}{\mathrm{A}_{\mathrm{S}}} \rho_{\text {ref }}\right) \cdot \mathrm{C}
$$

After infinite reflections, the flux sensed by the sensor, $\mathrm{J}_{\mathrm{src}}$, is

$$
\begin{gathered}
J_{\text {src }}=\frac{I_{\text {src }} A_{\text {src }}}{A_{s}} \rho_{\text {ref }} \rho+\frac{I_{\text {src }} A_{\text {src }}}{A_{s}} \rho_{\text {ref }} \rho^{2}+\frac{I_{\text {src }} A_{\text {src }}}{A_{s}} \rho_{\text {ref }} \rho^{3}+\ldots \ldots . . \\
J_{\text {src }}=\frac{I_{\text {src }} A_{\text {src }}}{A_{s}} \rho_{\text {ref }} \rho\left(\frac{1}{1-\rho}\right)
\end{gathered}
$$

Similarly, the flux sensed by the sensor from the reference beam, $\mathrm{J}_{\text {ref, }}$ is 


$$
J_{\text {ref }}=\frac{I_{\text {ref }} A_{\text {ref }}}{A_{S}} \rho^{2}\left(\frac{1}{1-\rho}\right)
$$

and the ratio 100 is

$$
100=J_{\text {src }} / J_{\text {ref }}=\frac{I_{\text {src }} A_{\text {src }} \rho_{\text {ref }}}{I_{\text {ref }} A_{\text {ref }} \rho}
$$

If a measurement is performed on a diffusely reflecting sample, the sample beam behaves like the $100 \%$ transmission test, except that the first reflection occurs with the samples reflectance, $\rho_{\text {smp. }}$. Equations A11 and A13b respectively become

$$
\begin{gathered}
G=I_{\text {src }} \frac{A_{\text {src }}}{A_{\mathrm{s}}} \rho_{\text {smp }} \\
J_{\text {src }}^{D}=\frac{I_{\text {src }} A_{\text {src }}}{A_{\mathrm{S}}} \rho_{\text {smp }} \rho^{2}\left(\frac{1}{1-\rho}\right)
\end{gathered}
$$

where $\mathrm{J}_{\text {src }}^{\mathrm{D}}$ is the flux sensed by the detector from the sample beam for a diffuse sample. If it is assumed that the reference beam does not change appreciably, the ratio $\mathrm{S}$ becomes

$$
\mathrm{S}=\mathrm{J}_{\mathrm{src}}^{\mathrm{D}} / \mathrm{J}_{\text {ref }}=\frac{\mathrm{I}_{\mathrm{src}} \mathrm{A}_{\text {src }} \rho_{\text {smp }}}{\mathrm{I}_{\text {ref }} \mathrm{A}_{\text {ref }}}
$$

Substituting Equations A15 and A18 into Equation 2, and with Z = 0, shows that the process indeed results in a measurement of $\rho_{\text {smp. }}$.

If a measurement is performed on a specularly reflecting sample, however, the result is again inaccurate. When the sample beam reflects from the sample, it is not distributed over the surface of the sphere. The reflection goes to the side of the sphere where it is then reflected over the entire surface of the sphere. It follows that after infinite reflections, the flux sensed by the detector from the sample beam for a specular sample, $\mathrm{J}_{\mathrm{src}}^{\mathrm{S}}$, is

$$
\begin{aligned}
& J_{\text {src }}^{S}=\frac{I_{\text {src }} A_{\text {src }} \rho_{\text {smp }}}{A_{s}} \rho^{2}+\frac{I_{\text {src }} A_{\text {src }} \rho_{\text {smp }}}{A_{S}} \rho^{3}+\frac{I_{\text {src }} A_{\text {src }} \rho_{\text {smp }}}{A_{s}} \rho^{4}+\ldots \ldots . \\
& J_{\text {src }}^{S}=\frac{I_{\text {src }} A_{\text {src }} \rho_{\text {smp }}}{A_{S}} \rho^{2}\left(\frac{1}{1-\rho}\right)
\end{aligned}
$$

and

$$
\mathrm{S}=\mathrm{J}_{\text {src }}^{\mathrm{S}} / \mathrm{J}_{\mathrm{R}}=\frac{\mathrm{I}_{\text {src }} \mathrm{A}_{\text {src }} \rho_{\text {smp }}}{\mathrm{I}_{\text {ref }} A_{\text {ref }}}
$$

Substituting Equations A15 and A20 into Equation 2, and with $\mathrm{Z}=0$, shows that an inequality exists. The estimation of $\rho_{\text {smp }}$ is actually $\rho_{\text {smp }} \rho$, and the calculation will underpredict by $2 \%$ (of the reading, not of full scale). By extension, measurements on a sample that is partially specular will have up to $2 \%$ error; depending on the directional distribution of reflected radiation. 


\section{Appendix B: Integrating Sphere Response to Errors due to Presence of Sample Holders.}

To gauge the sphere response (reduction in sensor strength) due to the presence of the sample holders, a worst case scenario was considered.

For the purpose of this discussion, a 4-surface model of the sphere is considered. Surface 1 is the opening of the sample holder. It was assumed to be a $15.75 \mathrm{~mm}$ diameter circle. Surface 2 is the first point of illumination from the sample beam on the sphere wall. It too was assumed to be a 15.75 diameter circle (for simplicity) with a reflectivity of $\rho=0.98$. Surface 3 is the outer surface of the sample holder. It was a $15.75 \mathrm{~mm}$ diameter tube that projects up to $30 \mathrm{~mm}$ into the sphere, with a reflectivity of $\rho_{3}$. Finally, Surface 4 is the integrating sphere surface. It has a diameter of $110 \mathrm{~mm}$ and a reflectivity of $\rho=0.98$.

We can perform a standard radiosity, J, balance on this geometry

$$
\begin{aligned}
& J_{1}=\rho_{1}\left[F_{11} J_{1}+F_{12} J_{2}+F_{13} J_{3}+F_{14} J_{4}\right] \\
& J_{2}=\rho\left[F_{21} J_{1}+F_{22} J_{2}+F_{23} J_{3}+F_{24} J_{4}\right]+I_{s r c} A_{\text {src }} \rho \\
& J_{3}=\rho_{3}\left[F_{31} J_{1}+F_{32} J_{2}+F_{33} J_{3}+F_{34} J_{4}\right] \\
& J_{4}=\rho\left[F_{41} J_{1}+F_{42} J_{2}+F_{43} J_{3}+F_{44} J_{4}\right]
\end{aligned}
$$

Here, the $F_{i j}$ 's are view factors between surfaces. Surfaces 1,2 , and 3 are not self viewing ( $F_{11}$ $\left.=F_{22}=F_{33}=0\right)$, and surface 1 and 3 and 2 and 3 cannot see one another $\left(F_{13}=F_{31}=0, F_{23}=\right.$ $\left.F_{32}=0\right)$. These view factors go to zero. Because surface 3 only views surface $4, F_{34}=1$. Further, $\rho_{1}=0$ making $\mathrm{J}_{1}=0$. Applying this to Equation B1 leaves

Combining

$$
\begin{aligned}
& \mathrm{J}_{2}=\rho\left[\mathrm{F}_{24} \mathrm{~J}_{4}\right]+\mathrm{I}_{\text {src }} \mathrm{A}_{\text {src }} \rho \\
& \mathrm{J}_{3}=\rho_{3}\left[\mathrm{~J}_{4}\right] \\
& \mathrm{J}_{4}=\rho\left[\mathrm{F}_{42} \mathrm{~J}_{2}+\mathrm{F}_{43} \mathrm{~J}_{3}+\mathrm{F}_{44} \mathrm{~J}_{4}\right]
\end{aligned}
$$

which can be rearranged to give

$$
\mathrm{J}_{4}=\rho\left[\mathrm{F}_{42}\left(\rho \mathrm{F}_{24} \mathrm{~J}_{4}+\mathrm{I}_{\text {src }} \mathrm{A}_{\text {src }} \rho\right)+\mathrm{F}_{43} \rho_{3} \mathrm{~J}_{4}+\mathrm{F}_{44} \mathrm{~J}_{4}\right]
$$

$$
\frac{J_{4}}{I_{\text {src }}}=\frac{F_{42} A_{\text {src }} \rho^{2}}{\left(1-F_{24} F_{42} \rho^{2}-F_{43} \rho_{3} \rho-F_{44} \rho\right)}
$$

Equation B4 is easily solved for a number of potential tube lengths. In doing so, it is revealed that if $\rho_{3}=0.05$ (black painted tube), the signal strength is reduced by about $60 \%$. For a more reflective tube with $\rho_{3}=0.85$, like the ones used in this work, the signal strength is reduced by about $20 \%$.

A more complex multi-surface version and a Monte-Carlo simulation of the integrating sphere were also developed. Both confirmed the results of this rudimentary model.

It is noted that the reference beam will also experience a reduction in detector signal strength due to the presence of the sample holder. Therefore, calibration with the sample holder in place will remove the influence of the sample holder on the measurement. 


\section{References}

[1] EnergyPlus, 2005. EnergyPlus Engineering Reference - The Reference to EnergyPlus Calculations, U.S. Department of Energy.

[2] ESP-r, 2008, Building Energy Simulation Code, University of Strathclyde, Glasgow, Available from: <http://www.esru.strath.ac.uk>

[3] Pedersen, C. O., Liesen, R.J., Strand, R.K., and Fisher, D.E., 2001, Toolkit for Building Load Calculations. Atlanta: American Society of Heating Refrigeration and Air Conditioning Engineers, Inc.

[4] Wright, J.L., Kotey, N.A., 2006, Solar Absorption by Each Element in a Glazing/Shading Layer Array, ASHRAE Transactions, Vol. 112, Pt. 2. pp. 3-12

[5] Kotey, N.A., Collins, M.R., Wright, J.L., and Jiang, T., 2009, A Simplified Method for Calculating Effective Solar Optical Properties of a Venetian Blind Layer for Building Energy Simulation ASME Journal of Solar Energy, Vol. 131(2).

[6] Kotey, N.A., Wright, J.L., Collins, M.R., 2009, A Detailed Model to Determine the Effective Solar Optical Properties of Draperies, ASHRAE Transactions, Vol. 115(1), .

[7] McCluney, R, 2006, Personal Communication, Florida Solar Energy Center.

[8] Hunn, B.D., Grasso, M.M., Beaudry, M.A., and P.J. House. 1991. Measurement of Bidirectional Solar Optical Properties of Shading Fabrics. ASHRAE Transactions, 97, pp. 455-464.

[9] Klems, J. H., 1994, A New Method for Predicting the Solar Heat Gain of Complex Fenestration Systems - 1. Overview and Derivation of the Matrix Layer Calculation, ASHRAE Transactions, Vol. 100 (1), pp. 1065-1072.

[10] Klems, J. H., 1994, A New Method for Predicting the Solar Heat Gain of Complex Fenestration Systems - 2. Detailed Description of the Matrix Layer Calculation, ASHRAE Transactions, Vol. 100 (1), pp. 1073-1086.

[11] Pettit, R.B., 1979, Hemispherical Transmittance Properties of Solar Glazings as a Function of Averaging Procedure and Incident Angle, Solar Energy Materials, 1: pp.125-140.

[12] Kotey, N.A., Wright, J.L. and Collins, M.R., 2009, Determining Off-Normal Solar Optical Properties of Drapery Fabrics, ASHRAE Transactions, 115(2), pp. xx-xx.

[13] Edwards, D.K, Gier, J.T., Nelson, K.E. and Roddick, R.D., 1961, Integrating Sphere for Imperfectly Diffuse Samples. Applied Optics, Vol. 51, pp. 1-12.

[14] Lovell, D.J., 1984, Theory and Applications of Integrating Sphere Technology. Laser Focus/Electro-Optics, pp. 86-96.

[15] ASTM E903-96. 1996. Standard Test Method for Solar Absorptance, Reflectance, and Transmittance of Materials Using Integrating Spheres. Philadelphia: American Society for Testing and Materials.

[16] ASTM E891-87. 1987. Standard Tables for Terrestrial Direct Normal Solar Spectral Irradiance for Air Mass 1.5. Philadelphia: American Society for Testing and Materials. 
[17] Keyes, M.W., 1967, Analysis and Rating of Drapery Materials Used for Indoor Shading. ASHRAE Transactions, Vol. 73(1).

[18] Kotey, N.A., Wright, J.L. and Collins, M.R., 2009, Determining Off-Normal Solar Optical Properties of Roller Blinds, ASHRAE Transactions, 115(1), pp. 145-154.

[19] Kotey, N.A., Wright, J.L. and Collins, M.R., 2009, Determining Off-Normal Solar Optical Properties of Insect Screens, ASHRAE Transactions, 115(1), pp. 155-164.

[20] Chakroun, W., Taylor, R. P., Steele, W. G., and Coleman, H.W., 1993, Bias Error Reduction Using Ratios to Baseline Experiments - Heat Transfer Case Study. Journal of Thermophysics and Heat Transfer, Vol. 7(4), pp. 754-757.

[21] Coleman, H.W., and Steele, W.G., 1999, Experimentation and Uncertainty Analysis for Engineers, $2^{\text {nd }}$ Edition, John Wiley \& Sons Inc. 